\title{
ROLE OF COMPUTED TOMOGRAPHY IN ASSESSING FLOOR OF THE MOUTH LESIONS
}

\author{
Mohamed A. Morsi Abdeldayem*, Omneya Gamal Eldin*, Nada salam* and Adel Ramadan**
}

\begin{abstract}
Background: The floor of the mouth (FOM) is located below the tongue in the oral cavity. Many diseases can occur in the FOM that are unique to this anatomical location. Clinical examination of the mucosal surface of the FOM is easy without imaging, because the superficial pathologies can be assessed by the bare eye. However, if the disease has a deep extension into the FOM or spreads beyond its boundaries, imaging may then be necessary. Different imaging modalities can be helpful in examining the FOM
\end{abstract}

Objective: To assess the role of CT in assessing lesions of the floor of the mouth with complementary US or MRI if needed.

Subjective: The study was carried out on 26 patients referred to the Radio-diagnosis department at Alexandria Main University Hospital diagnosed with floor of mouth lesions during the time period December 2018 to September 2020. Biphasic CT was performed in all 26 patients with an initial non contrast phase taken before contrast administration. Complementary MRI was done in 8 cases along with complementary ultrasound in 12 cases.

Results: MDCT helped in classifying lesions according to the nature of the lesion into three groups: cystic, solid and mixed lesions. Solid lesions were the commonest; fourteen $(53.8 \%)$ of the lesions were of solid nature, seven $(26.9 \%)$ were cystic in nature and lastly five $(19.2 \%)$ lesions were of mixed nature. Nineteen (73\%) lesions were well defined on MDCT, while seven (26.9\%) lesions showed ill-defined borders. The lesions were classified according to their pattern of enhancement into homogenous, heterogeneous, peripheral enhancement and non-enhancing lesions. Eleven $(42.3 \%)$ cases showed homogeneous enhancement, nine (34.6\%) showed heterogeneous enhancement, three (11.5\%) showed no enhancement, two (7.6\%) with peripheral enhancement and finally one $(3.8 \%)$ with enhancing septae.

Conclusion: $\mathrm{CT}$ is within reach with rapid image acquisition; this is why it is a primary investigation to broadly differentiate between different pathological processes in the floor of the mouth. Based upon the MDCT characteristics of the lesion such as tissue density, tissue composition, enhancement pattern and vascularity, MDCT helped to narrow the differential diagnosis.CT provides key information for proper staging of patients with oral cancer, such as the invasion depth, assessing if there is bone invasion and evaluation of lymph nodes.

* Department of Radiodiagnosis, Faculty of Medicine, Alexandria University

** Department of Maxillofacial and Plastic Surgery, Faculty of Dentistry, Alexandria University 


\section{INTRODUCTION}

Imaging has an important role in the assessment of deeper structures of the FOM preoperatively, besides providing vital information of local invasion and nodal involvement. Considering its worldwide accessibility, CT is a common diagnostic tool for assessment of FOM pathologies, occasionally as a substitute or complementing other imaging modalities. CT and MRI are the best modalities for FOM imaging; CT is readily available, while MRI has the upper hand in soft tissue resolution. ${ }^{(1)}$

The floor of the mouth (FOM) is situated in the oral cavity underneath the tongue. Many diseases can take place in the FOM that are unique to this anatomical location. Examination of the mucosa of the FOM clinically is easy without imaging, because the superficial pathologies can be assessed by the bare eye. However, if the disease has a deep extension into the FOM or spreads beyond its boundaries, imaging may then be necessary. Different imaging modalities can be supportive in examining the FOM. ${ }^{(2)}$

Diagnosis of lesions in FOM can be challenging diagnosis as various pathologies can take place in this space. The FOM variable types of tissue and specialized anatomical structures, this explains the occurrence of various systemic and local pathologies in this area. ${ }^{(3)}$

One of the limitations in MR imaging is artifacts produced by swallowing-related movement. With multidetector CT, imaging of the oral cavity and pharynx can be performed in a single short breath hold or even with the patient breathing normally. Patient movement artifact is virtually eliminated Volume acquisition of data allows image reconstruction in any desired plane. ${ }^{(4)}$

\section{AIM OF THE WORK}

The aim of this work was to assess the role of CT in assessing lesions of the floor of the mouth with complementary US or MRI if needed.

\section{PATIENTS AND METHOD}

The study was done on 26 patients referred to the Radio-diagnosis department at Alexandria Main University Hospital diagnosed with floor of mouth lesions during the time period December 2018 to September 2020. The patients included in this study must had a lesions extending into the FOM.

The excluded patients was; patients with poor renal function; patients allergic to contrast media; patients with lesions limited to the tongue and patients with unstable general condition.

\section{METHODS}

All patients with FOM lesions included in this study were subjected to the following:

- Thorough history taking: Detailed clinical history was taken including personal data and different systemic complaints.

- Review of previous imaging and clinical files.

- Renal function tests.

\section{Technique of MDCT neck}

\section{I- Patients/Image Acquisition}

\section{a) Patient preparation}

No specific patient preparation was requested except fasting for six hours prior to the procedure to avoid nausea and vomiting. No specific premedications were needed.

\section{b) Patient Positioning}

Supine position with neck hyperextended and shoulders lowered as much as possible. Patient motion should be avoided during the examination. Patient was instructed not to swallow nor cough as much as possible.

\section{c) Non contrast and contrast enhanced MDCT}

CT was performed using Siemens 64-detectors row helical scanner. 
CT was performed with the following technical parameters, gantry rotation time 0.75 second and gantry tilt is zero.

\section{II- Image Analysis/Interpretation:}

Using the workstation post-processing was done and images were evaluated by using dedicated analysis software. DICOM datasets were transmitted to a post processing workstation where $2 \mathrm{D}$ and $3 \mathrm{D}$ image processing was performed using open source software "Osirix".

\section{Statistical analysis of the data}

Data were fed to the computer and analyzed using IBM SPSS software package version 20.0. (Armonk, NY: IBM Corp) Qualitative data were described using number and percent. Quantitative data were described using range (minimum and maximum), mean, standard deviation.

\section{RESULTS}

The study included 26 patients with FOM lesions referred to the Radiology Department of Alexandria Main University Hospital, Faculty of Medicine, Alexandria University, seventeen of our patients was males (65.3\%) and nine females (34.6\%). Regarding patient"s age, patients in this study were classified with an age interval of 20 years. The patients were between one and 65 years with mean age of 37.29 years.Out of the seventeen males, twelve (70.5\%) were diagnosed to have benign lesions and five $(29.4 \%)$ were diagnosed to have malignant lesions. Out of the nine females, eight $(88.8 \%)$ were diagnosed to have benign lesions and one (11.1\%) was diagnosed with a malignant lesion. The most common patient's complaint was a FOM swelling which was found in nineteen cases $(73 \%)$ followed by pain in seventeen cases $(65.3 \%)$, hoarseness of voice in two cases (7\%), trismus in one case $(3.8 \%)$, otalgia in one case $(3.8 \%)$, sore throat in one case $(3.8 \%)$, breathing difficulty in one case $(3.8 \%)$ and dysphagia in one case (3.8\%).The cases were clas- sified according to their anatomical site into unispatial and multispatial lesions. Unispatial (respecting the spatial distribution) lesions were twelve (46.1\%) of the lesions, involving one space. Multiple lesions that involved more than one space but still respected the spatial distribution were considered as unispatial lesions. These included four cases with multiple stones, one case of venous malformation and one case of two SL necrotic lymph nodes.

Multispatial lesions (did not respect the spatial distribution) were fourteen (53.8\%) of the lesions, eleven $(78.5 \%)$ of them occupied more than two anatomical spaces, while three $(21.4 \%)$ of them occupied two spaces. Those who involved more than two spaces were five out of six $(83.3 \%)$ of the inflammatory lesions in the current study (plunging ranula, two cases of FOM abscesses and two cases of FOM cellulitis), five out of seven $(71.4 \%)$ of the vascular. While those limited to two spaces were: FOM abscess, SCC of the FOM and lymphomatous node.

The majority of lesions did not cross the midline; only six $(23 \%)$ lesions crossed the midline; those were plunging ranula, FOM abscess, venous malformation, hemangiomas, lingual artery pseudoaneurysm and SCC of the FOM. While the remaining twenty (76.9\%) lesions respected the midline. Those were four out of six (66.6\%) inflammatory lesions (FOM abscess, cellulitis in FOM and SM retention cyst), all (100\%) cases with stones in FOM, four out of seven $(57.1 \%)$ vascular lesions (venous malformation, lymphatic malformations and venolymphatic malformation), neurofibromas and five out of six (83.3\%) malignant neoplastic lesions (FOM carcinoma, SL necrotic lymph node, neoplastic SM lymph node, retromolar trigonce cancer and lymphomatous lymph node).

MDCT helped in classifying lesions based upon the nature of the lesion into three groups: cystic, solid and mixed lesions. Solid lesions were the commonest; fourteen (53.8\%) lesions were of solid nature, seven $(26.9 \%)$ were cystic in nature and lastly 
five (19.2\%) lesions were of mixed nature.

Lesions were further classified based upon their borders into well-defined and ill-defined lesions on MDCT. Nineteen (73\%) lesions were well defined on MDCT, while seven (26.9\%) lesions showed illdefined borders.The lesions were classified based upon their pattern of enhancement into homogenous, heterogeneous, peripheral enhancement and non-enhancing lesions.

In this study, the FOM lesions were classified into inflammatory lesions, stones, vascular lesions, benign neoplastic lesions and malignant neoplastic lesions.

Clinical suspicion coupled with MDCT were the mainstay in reaching the final diagnosis. However, further investigations were needed for confirmation. In inflammatory lesions, plunging ranula and SM retention cysts were confirmed by excisional biopsy, while FOM abscesses were confirmed by pus upon surgical drainage.

In case of neurofibroma, excisional biopsy was needed to confirm the diagnosis. As for the FOM carcinomas, incisional biopsy was done. Finally, fine needle aspiration cytology followed by core needle biopsy were done in neoplastic lymph nodes. Vascular lesions in the FOM were clinically suspected and confirmed radiologically with no need for further investigations.

\section{Imaging findings of the inflammatory lesions in floor of mouth $(n=6)$}

In this study, two (33.3\%) out of six patients were diagnosed as FOM abscesses, two (33.3\%) were diagnosed as FOM cellulitis, one (20\%) as SM retention cyst and one (20\%) as plunging ranula by contrast enhanced CT. Four $(66.6 \%)$ were males and two $(33.3 \%)$ were females.

The CT tissue density of the two (33.3\%) abscesses was of fluid density, showing a peripherally enhancing thick wall with necrotic center. Similarly two (33.3\%) cases showed fluid density (SM retention cyst and plunging ranula), however with a thin peripheral smooth wall and no enhancing soft tissue components. Whereas two $(33.3 \%)$ cases had soft tissue density (Cellulitis in FOM secondary to periapical cyst and cellulitis secondary to mandibular osteomyelitis, the latter also showed a solid periosteal reaction). Based upon the enhancement pattern, the abscesses (33.3\%) had a capsular ring enhancement while the FOM cellulitis $(33.3 \%)$ showed mild diffuse enhancement. As for the retention cyst (16.6\%),

TABLE (1): Enhancement pattern in the studied group $(n=26)$

\begin{tabular}{|c|c|c|}
\hline Enhancement pattern & No & $\%$ \\
\hline Homogenous enhancement & 11 & 46.1 \\
\hline Cellulitis & 2 & 7.6 \\
\hline Capillary hemangioma & 1 & 3.8 \\
\hline Neurofibromas & 1 & 3.8 \\
\hline Venous malformation & 1 & 3.8 \\
\hline $\begin{array}{l}\text { Stones associated with homogeneous SM } \\
\text { gland enhancement }\end{array}$ & 6 & 23 \\
\hline Heterogeneous enhancement & 9 & 30.7 \\
\hline Venous malformation & 1 & 3.8 \\
\hline Veno-lymphatic malformation & 1 & 3.8 \\
\hline Lingual artery pseudo-aneurysm & 1 & 3.8 \\
\hline FOM carcinoma & 2 & 7.6 \\
\hline $\begin{array}{l}\text { Recurrent lingual cancer with SL necrotic } \\
\text { lymph node }\end{array}$ & 1 & 3.8 \\
\hline Neoplastic SM lymph node & 1 & 3.8 \\
\hline Retromolar trigone cancer & 1 & 3.8 \\
\hline Matted lymph node in lymphoma & 1 & 3.8 \\
\hline Peripheral enhancement & 2 & 7.6 \\
\hline FOM abscess & 2 & 7.6 \\
\hline Enhancing septae & 1 & 3.8 \\
\hline SM retention cyst & 1 & 3.8 \\
\hline Non enhancing & 3 & 15 \\
\hline Plunging ranula & 1 & 3.8 \\
\hline Lymphangioma & 2 & 7.6 \\
\hline
\end{tabular}


it showed enhancement of its internal septae only. Finally, the plunging ranula $(16.6 \%)$ showed no enhancement after contrast administration.

Five $(83.3 \%)$ cases showed related fat stranding, while one $(16.6 \%)$ case, plunging ranula, showed no associated fat stranding. Only (16.6\%) one case, FOM abscess, showed internal air foci as well as a SM cutaneous fistula.

The CT helped in detection of a periapical cyst in three (50\%) cases (abscesses and cellulitis), that was accused to be the infection source in such cases.

Plunging ranula was shown as a bi-lobed well defined cystic lesion having no solid components,

TABLE (2): Final diagnosis of lesions in the studied group $(n=26)$

\begin{tabular}{|c|c|c|}
\hline Lesions according to final diagnosis & $\begin{array}{c}\text { Num- } \\
\text { ber }\end{array}$ & $\%$ \\
\hline Inflammatory lesions & 6 & 23 \\
\hline Plunging ranula & 1 & 3.8 \\
\hline Abscess & 2 & 7.6 \\
\hline Cellulitis & 2 & 7.6 \\
\hline SM retention cyst & 1 & 3.8 \\
\hline Stones & 6 & 23 \\
\hline Vascular lesions & 7 & 26.9 \\
\hline Venous malformation & 2 & 7.6 \\
\hline Capillary hemangioma & 1 & 3.8 \\
\hline Lymphatic malformation & 2 & 7.6 \\
\hline Veno-lymphatic malformation & 1 & 3.8 \\
\hline Lingual artery pseudoaneurysm & 1 & 3.8 \\
\hline Benign lesions & 1 & 3.8 \\
\hline Neurofibroma & 1 & 3.8 \\
\hline Malignant lesions & 6 & 23 \\
\hline FOM carcinoma & 2 & 7.6 \\
\hline Recurrent lingual cancer & 1 & 3.8 \\
\hline Neoplastic lymph node & 2 & 7.6 \\
\hline Retromolar trigone cancer & 1 & 3.8 \\
\hline
\end{tabular}

arising from the left SL space above the mylohyoid muscle and crossing inferiorly to the right SL and SM spaces. (Fig.1) It showed no post-contrast enhancement after contrast administration.

In FOM abscesses, one case showed an irregular fluid density collection involving submental and both SL spaces. The other case showed a right SM mixed fluid and gas density collection, that was seen extending superiorly to the right masticator space and inferiorly to the submental space, and subcutaneously to the mediastinum leading to mediastinitis. In the latter, a SM subcutaneous fistula as well as a periapical cyst that was seen associated with cortical erosion of the alveolar margin of the mandible were noted. Both cases showed peripheral capsular enhancement after contrast administration, confirming their diagnosis. (Fig.2)

In FOM cellulitis, first case showed an ill-defined phlegmon obscuring the left SL space along with subcutaneous fat stranding and thickening of the related platysma muscle. It showed mild homogeneous post contrast enhancement. Enlarged reactive loco-regional lymph nodes were also noted. A peri-apical cyst and cortical erosion of the alveolar mandibular margin, that was the source of the infection, were also noted. (Fig. 3).

Second case of FOM cellulitis showed generalized increased bone density (osteopetrosis) with secondary left mandibular osteomyelitis, together with a solid periosteal reaction. This was seen in association with an ill-defined homogeneously enhancing tissue (cellulitis) surrounding the mandibular ramus extending on both lingual and buccal surfaces. It is seen medially abutting the left myelohyoid muscles and posteriorly displacing the left SM gland. Enlarged reactive loco-regional lymph nodes were also noted. (Fig. 4)

As for the SM retention cyst, CT showed a welldefined, thin walled cystic lesion at the anatomical site and near totally replacing the SM gland. It showed fine enhancing internal septae with a mildly enhancing wall. (Fig. 5) 


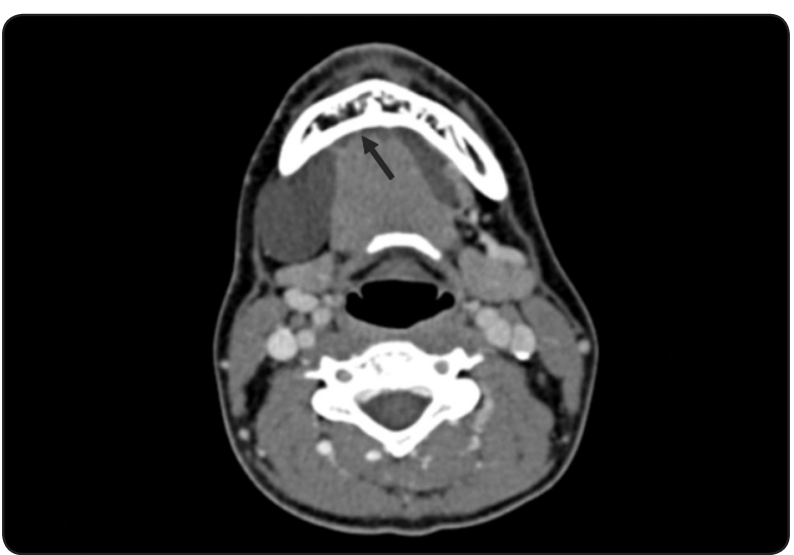

Fig. (1): Axial contrast enhanced MDCT for a pathologically proven plunging ranula showing a bilobed well defined cyst in the left SL space, crossing the midline (red arrow) to the right SL and SM spaces.
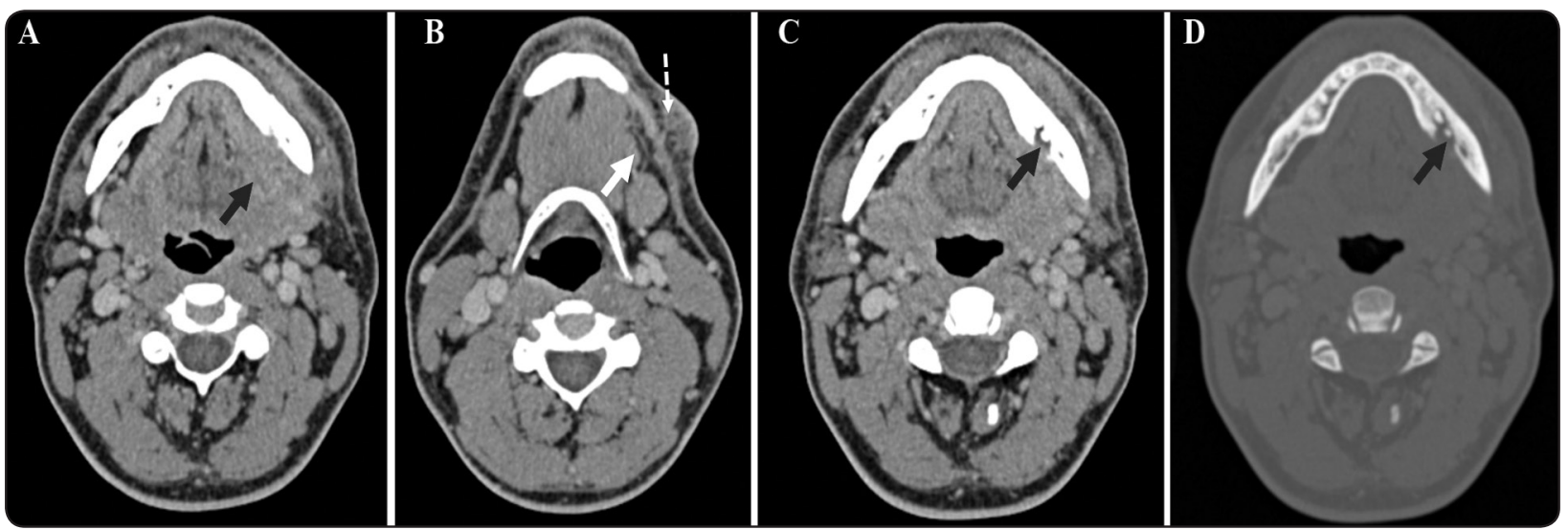

Fig. (3): A,B,C \& D axial contrast enhanced MDCT of FOM cellulitis, showing (A) an ill-defined left sublingual phlegmon (red arrow), along with (B) subcutaneous fat stranding (dashed white arrow) and thickening of the platysma (white arrow). (C $\& D)$ An associated left periapical cyst is noted with (D) cortical erosion of the alveolar margin of the mandible (Red arrows).

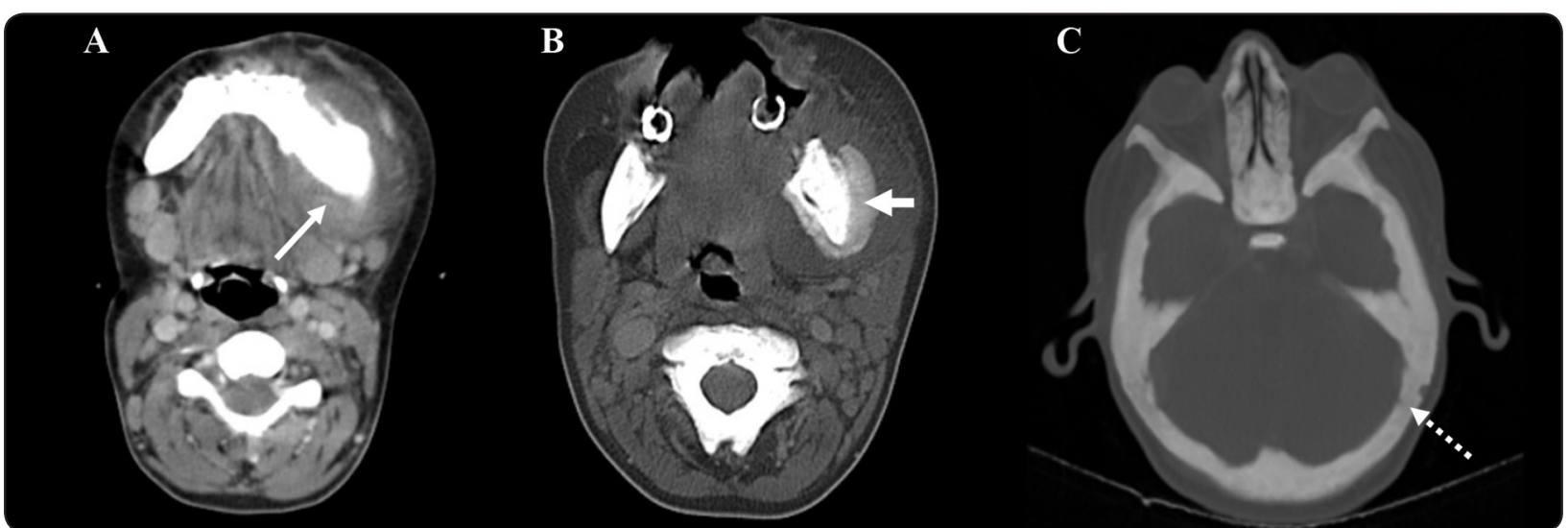

Fig. (4): A, B \&C axial contrast enhanced MDCT; soft tissue and bone window, of a case of mandibular osteomyelitis secondary to osteopetrosis showing (A) an ill-defined homogeneously enhancing tissue (cellulitis) surrounding the mandibular ramus extending on both buccal and lingual surfaces (red arrow). It is seen medially abutting the left myelohyoid muscles and posteriorly displacing the left SM gland. (B) Dual window showing a solid periosteal reaction around the left mandibular ramus (white arrow). (C) Diffuse increased bone density noted in the calvarium. (Red dotted arrow) 


\section{Imaging findings of stones in floor of mouth $(n=6)$}

At the current study, 6 out of 26 patients (23\%) were diagnosed as stones in the FOM. Three (50\%) were males and three $(50 \%)$ were females.

More than one stone (two stones) was detected in two $(33.3 \%)$ cases, while four $(66.6 \%)$ cases only showed one stone.

CT detected apparent sialectasis in two (33.3\%) cases (Fig.6, 7), one was secondary to a stricture that was evident on complementary MRI.

As for associated gland inflammation (sialadenitis), three (50\%) cases were associated with sial-

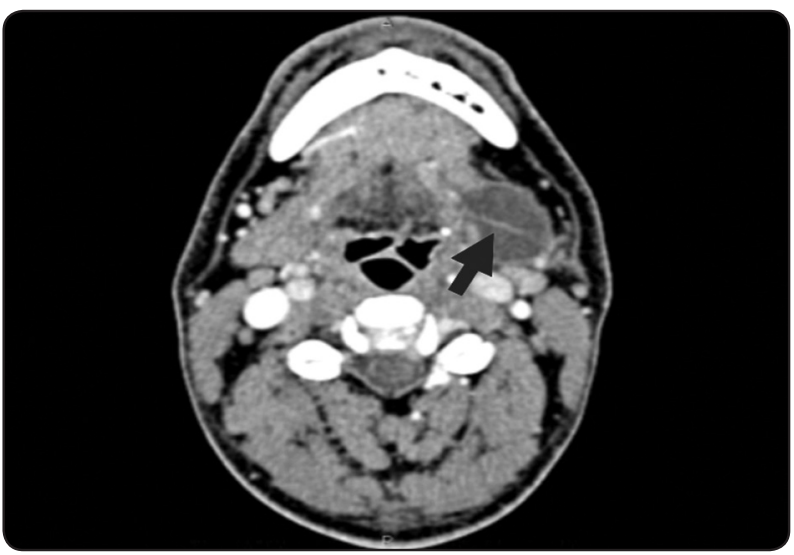

Fig. (5): Axial contrast enhanced MDCT of a left SM retention cyst, seen near totally replacing the left SM gland with internal enhancing septa (red arrow) and a mildly enhancing wall.

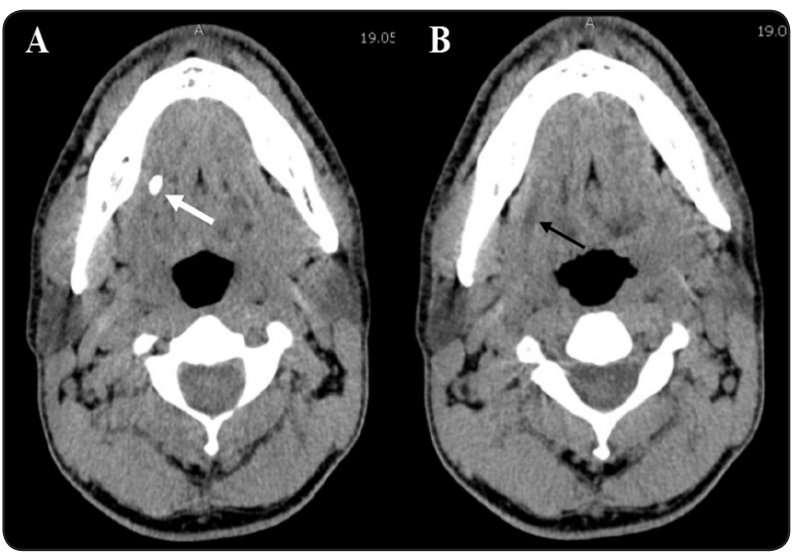

Fig. (6): A \& B axial non contrast MDCT of the same patient showing (A) a right SM duct stone (white arrow) with (B) upstream sialectasis (red arrow).
TABLE (3): MDCT findings in the inflammatory lesions in the studied group were as follow: $(n=6)$

\begin{tabular}{lll}
\hline \multicolumn{1}{c}{ Imaging findings } & No. & Percent $(\boldsymbol{\%})$ \\
\hline CT tissue density & & \\
\hline Soft tissue density & 2 & 33.3 \\
\hline Fluid density & 2 & 33.3 \\
\hline Mixed & 2 & 33.3 \\
\hline Bone density & 1 & 16.6 \\
\hline Enhancement & & \\
\hline Peripheral enhancement & 2 & 33.3 \\
\hline Homogeneous enhancement & 2 & 33.3 \\
\hline Enhancing septae & 1 & 16.6 \\
\hline Non enhancing & 1 & 16.6 \\
\hline Fat stranding & 5 & 83.3 \\
\hline Internal air foci & 1 & 16.6 \\
\hline Associated fistula & 1 & 16.6 \\
\hline Periapical cyst & 3 & 50 \\
\hline
\end{tabular}

*More than one finding was detected in the same patient.

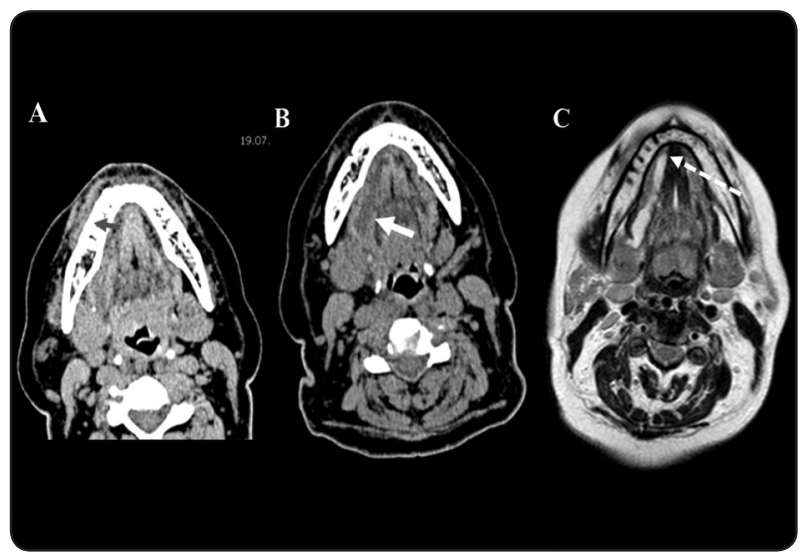

Fig. (7): A \& B axial non contrast MDCT of the same patient showing (A) a right SM duct stone (red arrow) with (B) downstream sialectasis (white arrow) due to a stricture that was a result of previous intervention. (C) Axial T2 MRI showing stricture of the right SM duct (white dashed arrow). 
adenitis. Two (33.3\%) showed acute inflammation with related fat stranding and one $(16.6 \%)$ showed chronic inflammation manifested as SM gland atrophy with fat replacement.

\section{Imaging findings of vascular lesions in floor of mouth $(n=7)$}

At the current study, 7 out of 26 patients (26.9\%) were diagnosed as vascular lesions (lymphatic malformation, capillary hemangioma, venous malformation, veno-lymphatic malformation and lingual artery pseudoaneurysm) on MDCT. Two (28.5\%) of the patients who presented with vascular lesions were females and five (71.4\%) were males.Three $(42.8 \%)$ of them showed soft tissue density on MDCT (venous malformation and capillary hemangioma) and two (28.5\%) were fluid density (lymphatic malformation). While one (14.2\%) was of mixed density (veno-lymphatic malformation) and another one (14.2\%) showed initial hyperdensity (lingual artery pseudoaneurysm).

Regarding the enhancement pattern, two (28.5\%) cases showed homogeneous post contrast enhancement (capillary hemangioma and venous malformation) and three $(42.8 \%)$ cases showed heterogeneous post contrast enhancement (venous malformation, veno-lymphatic malformation and lingual artery pseudoaneurysm), while the remaining two (28.5\%) cases were non enhancing (lymphatic malformations). As for the borders of the lesions, two (28.5\%) cases had ill-defined borders (lingual artery pseudoaneurysm and venolymphatic malformation), while the remaining cases $(71.4 \%)$ had well-defined borders.Only one (14.2\%) case, lymphatic malformation, was unispatial (in left SM space) while the remaining six $(85.7 \%)$ cases were multispatial, occupying 2 or more anatomical spaces.

CT helped demonstrate the presence of phleboliths (which are pathognomonic for venous malformation) in three $(42.8 \%)$ cases (venous malformations and veno-lymphatic malformation).
Venous malformations appeared as well-defined soft tissue lesions, showing phleboliths on non contrast $\mathrm{CT}$, confirming the venous malformations diagnosis. First case showed a lobulated soft tissue lesion in the submental, SL and SM spaces. Upon contrast administration, it showed a heterogeneous enhancement. The other case presented as multiple soft tissue lesions, with involvement of the right SL and SM space. Upon contrast administration, it showed homogeneous post contrast enhancement of its lesions. Further complementary MRI was carried out to support the diagnosis.

In capillary hemangioma, CT helped identifying a well-defined multispatial soft tissue lesion involving both SL spaces and the lingual septum. No phleboliths were seen. Upon contrast administration, marked homogenous enhancement was noted. CT also helped identifying other similar lesions in both parapharyngeal spaces, laryngeal space and prevascular mediastinal space.

As for lymphatic malformations, the two cases appeared as homogeneously cystic lesions with no detectable intrinsic septations. First case showed the lymphangioma located at the left SM and SL space. It showed mass effect in the form of posterior abutment and displacement of the left SM gland with lateral displacement of the platysma muscle. The other case was located in the left SM space with mild abutment of the SM gland medially. Both cases did not enhance after contrast administration. On CT, veno-lymphatic malformation appeared as an ill-defined mixed cystic and solid swelling in the right SM, SL spaces and the right paraglottic fat. It showed a small phlebolith and after contrast administration, heterogeneous enhancement of its soft tissue component was noted.

Finally, lingual artery pseudoaneurysm occurred as a post-operative complication, patient suffered from mandibular cancer and underwent hemi-mandibelectomy. Later, he had a rapidly growing pulsating mass which appeared on $\mathrm{CT}$ as an ill-defined initially hyperdense space occupying mass in the 
FOM, mainly involving the right SL space, submental space, right masticator space, root of the tongue and the surgical bed of hemi-mandibelectomy. It is seen mildly crossing the midline and superiorly displacing the tongue. It showed marked post contrast enhancement with a surrounding peripheral non enhancing thrombus. Its connection to the eccentrically dilated lingual artery can be well visualized on the three planes. This was seen associated with diffuse subcutaneous fat stranding.

TABLE (4): MDCT findings in in vascular lesions in the studied group were as follow: $(n=7)$

\begin{tabular}{lll}
\hline Imaging findings & No. & Percent (\%) \\
\hline CT Density & 3 & $42.8 \%$ \\
\hline Soft tissue density & 2 & $28.5 \%$ \\
\hline Fluid density & 1 & $14.2 \%$ \\
\hline Mixed (soft tissue and fluid) & 1 & $14.2 \%$ \\
\hline Initial hyperdensity & & \\
\hline Enhancement & 2 & $28.5 \%$ \\
\hline Homogeneously enhancing & 3 & $42.8 \%$ \\
\hline Heterogeneously enhancing & 2 & $28.5 \%$ \\
\hline Non enhancing & & \\
\hline Borders & 5 & $71.4 \%$ \\
\hline Well defined & 2 & $28.5 \%$ \\
\hline Ill defined & & \\
\hline Spatial distribution & 1 & $14.2 \%$ \\
\hline Unispatial & 6 & $85.7 \%$ \\
\hline Multispatial & 3 & $42.8 \%$ \\
\hline Phleboliths & & \\
\hline
\end{tabular}

Imaging findings of the benign neoplastic lesions in floor of mouth $(n=1)$

In this study, one male out of 26 patients (3.8\%) was diagnosed having a benign neoplastic lesion (neurofibromas) by contrast enhanced MDCT that was further confirmed by histopathology.

On CT they were shown to be multiple multispatial well-defined soft tissue lesions that did not cross the midline, notably at left SM space, left SL space, left masticator space, left buccal space, left parotid region, left parietal region subcutaneously and left retro-auricular. After contrast administration, they showed mild homogeneous post contrast enhancement.

\section{Imaging findings of the malignant neoplastic le- sions in floor of mouth $(n=6)$}

In this study, six out of 26 patients (23\%) were diagnosed as malignant neoplastic lesions by contrast enhanced MDCT and were further confirmed by histopathology. Five (83.3\%) cases were males and one $(16.6 \%)$ was a female with age ranging from 36 years (retromolar trigone cancer) to 65 years (supraglottic cancer with metastatic lymph node in the left SM space).

Regarding the CT tissue density and enhancement pattern, all (100\%) cases showed mixed density (soft tissue and necrosis) and heterogeneous enhancement with areas of breakdown.

Regarding the borders of the malignant neoplastic lesions, five $(83.3 \%)$ cases had ill-defined borders while one $(16.6 \%)$ case had well defined borders. However, concerning spatial distribution, three $(50 \%)$ cases were multispatial and three $(50 \%)$ cases were unispatial.

The majority of these lesions had ill-defined borders $(83 \%)$ and heterogeneous enhancement. Conversely, most of the benign lesions had well defined borders (90\%) and homogeneous enhancement. The $\mathrm{CT}$ demonstrated bony changes in two $(33.3 \%)$ ) cases; one (16.6\%) case (retromolar trigone cancer) showed bony invasion with cortical disruption and another case (16.6\%) showed related mandibular sclerosis (FOM carcinoma). The remaining four $(66.6 \%)$ cases showed no significant associated bone changes.

As regards to peri-tumoral invasion, all (100\%) were found involving more than one muscle. Four $(66.6 \%)$ of them were shown to invade the myelohyoid muscle, three $(50 \%)$ invaded the genioglos- 
sus muscle and two (33.3\%) invaded the geniohyoid muscle. One (16.6\%) case invaded the pterygoid muscles and another one (16.6\%) invaded the masseter muscle. Invasion of the SM gland was also noted in one (16.6\%) case.

Three $(50 \%)$ of the malignant lesions showed neoplastic nodes. First case, an ill-defined heterogeneously enhancing left SM metastatic lymph node (level Ib) was noted with internal areas of breakdown. It showed extracapsular extension and was seen inseparable from the overlying platysma. It was also shown superiorly displacing the left SM gland and posteriorly abutting the left sternomastoid muscle.

The second case showed two small well defined heterogeneously enhancing metastatic lymph nodes with necrotic center in the right SM space (level Ib) and anterior to the right internal jugular vein (level IIa). The latter was seen mildly indenting the posterior aspect of the right SM gland anteriorly.

The last case was a lymphomatous lymph node, that was shown on CT as a well defined matted lymph node in the right SM and right masticator spaces, slightly extending into the anterior cervical space. It showed mild heterogeneous enhancement with necrotic areas after contrast administration. It was also seen infiltrating the overlying platysma, right SM gland and the right masseter muscle.

As for squamous cell carcinoma of the FOM, both cases were seen as ill-defined heterogeneously enhancing soft tissue lesions. First case was seen limited to the right SL space; did not cross the midline. It was shown to infiltrate the right mylohyoid and the right genioglossus muscle. While the other case was seen crossing the midline to involve both sublingual spaces. It infiltrated the anterior aspect of both genioglossus and mylohyoid muscles sparing the geniohyoid muscles. The latter was seen associated with bilateral SM duct dilatation (secondary to obstruction by the carcinoma). No associated bony changes was detected on CT in both cases.
Retromolar trigone cancer appeared on CT as an ill-defined heterogeneously enhancing soft tissue lesion epicentered on the right retromolar trigone, extending anteriorly to the right SL space, obliterating its fat planes with infiltration of the right mylohyoid muscle and abutment with no definite infiltration of the right genioglossus muscle. Posteriorly extending into right masticator space, infiltrating the lateral and medial pterygoid muscles and the glossotonsillar sulcus. Laterally invading the mandibular bone,

TABLE (5): MDCT findings in malignant neoplastic lesions in the studied group were as follow: $(n=6)$

\begin{tabular}{|c|c|c|}
\hline Imaging findings & No. & Percent $(\%)$ \\
\hline \multicolumn{3}{|l|}{ CT tissue density } \\
\hline Mixed tissue density & 6 & $100 \%$ \\
\hline \multicolumn{3}{|l|}{ Enhancement pattern } \\
\hline Heterogeneous enhancement & 5 & $83.3 \%$ \\
\hline Homogeneous enhancement & 1 & $16.6 \%$ \\
\hline \multicolumn{3}{|l|}{ Borders } \\
\hline Well defined & 1 & $16.6 \%$ \\
\hline Ill defined & 5 & $83.3 \%$ \\
\hline \multicolumn{3}{|l|}{ Spatial distribution } \\
\hline Unispatial & 3 & $50 \%$ \\
\hline Multispatial & 3 & $50 \%$ \\
\hline \multicolumn{3}{|l|}{ Bony changes } \\
\hline Bone sclerosis & 1 & $16.6 \%$ \\
\hline Bone invasion & 1 & $16.6 \%$ \\
\hline No bony changes & 4 & $66.6 \%$ \\
\hline \multicolumn{3}{|l|}{ Peritumoral invasion } \\
\hline Myelohyoid muscle & 4 & $66.6 \%$ \\
\hline Genioglossus muscle & 3 & $50 \%$ \\
\hline Geniohyoid muscle & 2 & $33.3 \%$ \\
\hline Pterygoids & 1 & $16.6 \%$ \\
\hline Masseter muscle & 1 & $16.6 \%$ \\
\hline Submandibular gland & 1 & $16.6 \%$ \\
\hline
\end{tabular}

*More than one finding was detected in the same patient. 
including the body and ramus of the mandible. Medially, obliterating the right parapharyngeal space and invading the right tonsil. On complementary MRI axial STIR sequence, it showed a high signal intensity lesion at the right retromolar trigone with the above mentioned extensions.

As a final observation, malignant lesions shared common CT features including an ill-defined border with heterogeneous enhancement and areas of necrosis. Conversely, benign lesions showed well defined borders (apart from cellulitis in FOM), rarely showed heterogeneous enhancement (only TABLE (6): Pathologies and their ultrasound findings noted in venous malformation, veno-lymphatic malformation and lingual artery pseudoaneurysm) without necrosis except for one case of FOM abscess. In addition, malignant lesions were solid in nature, while benign lesions varied between cystic and solid nature.

\section{Complementary US and MRI}

US was done in twelve cases; four of the inflammatory lesions (Plunging ranula, FOM abscess, two cases of FOM cellulitis), six cases with SM duct stones and two cases with vascular lesions.

\begin{tabular}{ll}
\hline Pathology & Ultrasound findings \\
\hline Plunging Ranula & $\begin{array}{l}\text { A thin walled cystic lesion in the right SM and SL spaces with thin extension into the left } \\
\text { SL space }\end{array}$ \\
\hline FOM abscess & An irregular cyst like lesion in submental and both SL spaces with mild turbid content \\
\hline FOM cellulitis & Enlarged reactive looking deep cervical lymph node \\
\hline $\begin{array}{l}\text { FOM cellulitis secondary to } \\
\text { mandibular osteosarcoma }\end{array}$ & Enlarged reactive looking SM lymph node \\
\hline SM duct stones & Intraductal hyperechoic lines accompanied with posterior acoustic shadowing. \\
\hline SM duct stricture & Dilatation of the right SM duct with no definite obstructive lesion \\
\hline Capillary hemangioma & A SL vascular lesion showing no evidence of arterial communication \\
\hline Lymphangioma & $\begin{array}{l}\text { A macrocystic lesion in the left SM space with minor extension into the left SL space. No } \\
\text { solid components nor internal vascularity were detected. }\end{array}$ \\
\hline
\end{tabular}

Different complementary MRI sequences were used in eight cases; axial T1-weighted sequence was done once in a patient with SM duct stone and stricture. Likewise, sagittal T2-weighted and coronal T2-weighted sequences were performed once in a case of SL necrotic lymph node.

\section{DISCUSSION}

This study consisted of 26 patients, including 17 males (65.3\%) and nine females (34.6\%) with ages ranging from 1-65 years with mean age 37.29 years old. Out of the 26 lesions in the FOM in our study, six (23\%) were malignant while 20 (76.9\%) were of benign nature. Similarly, Ying Yuan et al, ${ }^{(5)}$ found that males are more affected than females with a percentage of $82.9 \%$ to $17 \%$. Conversely, our study differed from Ying Yuan et al,.(5) who had a higher percentage of malignant lesions $(86.5 \%)$ in comparison to benign lesions (13.4\%). This is mostly due to the small sample size in our study.

The range of symptoms we had in the current study included swelling in the FOM which was found in 18 cases $(68 \%)$ followed by pain in 11 
cases $(32 \%)$, hoarseness of voice in 2 cases (7\%), trismus in 1 case ( $4 \%$ ), otalgia in 1 case $(4 \%)$, sore throat in 1 case (4\%) and breathing difficulty in 1 case $(4 \%)$. This was similar to Ahmed et al, ${ }^{(6)}$ who reported that the most common symptom in their cases was a swelling in the FOM followed by pain.

Our study had six cases of inflammatory lesions in the FOM. Three of which had a previous odontogenic infection. The three lesions were noted to be multispatial, involving the SM space and SL space equally and to a lesser extent the submental and parapharyngeal space. This matched Rega et al, ${ }^{(7)}$ who stated in his study that multiple space infections are more encountered than single space infections in head and neck patients presenting with infections of odontogenic origin.

The prevalence of simple ranulas is 0.2 cases for every 1000 people. The prevalence for plunging ranulas is unknown, but expected to be significantly lower. Charnoff et al. ${ }^{(8)}$ described the plunging ranula on $\mathrm{CT}$ as a large uni-locular fluid density mass with a smooth capsule and lacking internal septations. This agreed with the present study in lacking internal septations, however, it disagreed in being a bilocular cystic collection rather than being unilocular. Some plunging ranula cases were also reported secondary to sialolith removal, transpositioning of the duct and implant placement. Lastly, a duct from the SL gland may unite with the SM gland or its duct, forming ranulas continuous with the SM gland. ${ }^{(9)}$

Plunging ranulas most commonly occupy the SM triangle and connect with the SL gland by crossing over the posterior edge of mylohyoid muscle. Coit et $\mathrm{al},{ }^{(10)}$ reached a conclusion that diagnosing a plunging ranula was greatly suggested by the "tail sign', a small extension into the SL space with the majority of the cyst seen in the SM space. This was different from our study, as our case of plunging ranula did not show the tail sign, but rather was epicentered upon the SM and SL spaces. This might also be due to the previously explained etiologies of plunging ranulas and the small sample size of the present study.

At the present study, six out of 26 cases (23\%) were diagnosed with sialolithiasis, two out of six cases (33.3\%) involved the SM duct and four out of six $(66.6 \%)$ involved the SM gland and the SM duct. This was matching with Laine et al, ${ }^{(11)}$ who stated that sialolithiasis is most commonly encountered in the SM gland (60\%-90\% of cases) followed by parotid glands $(10 \%-20 \%$ of cases). This is explained by the high mucus content and viscous nature of the SM gland secretions, making it more prone to calculous disease.

Out of the six patients diagnosed with sialolithiasis, two $(33.3 \%)$ were noted in the mid portion of the SM duct and four (66.6\%) were noted at the posterior border of the SM duct. This was similar to Madani et al ${ }^{(12)}$ who also stated that a great number of stones (85\%) occur in the SM duct:30\% near the ductal orifice, $20 \%$ in the mid-duct and $35 \%$ at the bend in the duct at the posterior border of the mylohyoid muscle.

La'porte et al, ${ }^{(13)}$ reported that low-flow venous malformations occurring in the head and neck are frequently observed in the buccal space or the FOM and may extend to involve SL or SM spaces. This was observed in three $(100 \%)$ cases with venous malformation and veno-lymphatic malformation in our study, where the three cases were seen in the FOM involving the SL and SM glands.

Harnsberger et al, ${ }^{(14)}$ described venous malformations on CT as lobulated or multilobulated lesions, isodense to muscle, that can infiltrate multiple spaces in the head and neck. This agreed with the present study, where one $(50 \%)$ out of two patients diagnosed with venous malformations, showed a multi-lobulated appearance involving the submental space and bilateral SL and SM spaces. While the other case showed multiple discrete well defined lesions involving right SL and SM spaces, right upper lip, right parotid, left oropharyngeal wall, left masseter muscle, left temporalis muscle, right prevertebral space at DV1 and subcutaneously in the scalp. 
Meesa et al, ${ }^{(15)}$ as well as Harnsberger et al, ${ }^{(14)}$ also stated that $\mathrm{CT}$ is a priceless modality in diagnosing venous malformations, as it can identify small calcified phleboliths, it can also reveal bone remodeling close to the lesion. This coincided with the present study, as two (100\%) cases diagnosed with venous malformations along with one (100\%) case diagnosed with veno-lymphatic malformation showed calcified phleboliths. However, it disagreed with Harnsberger et $\mathrm{al}^{\left({ }^{(14)}\right.}$ in which none of the above mentioned cases showed bone remodeling close to the lesion, likely due to the lesion location away from the bone.

In this study, two (100\%) cases diagnosed with LMs, were found in the SM space. This went along with Navarro et al, ${ }^{(16)}$ who stated that LMs can exist anywhere where lymphatic tissue exists, but nearly $75 \%$ of lesions are in the region of head and neck. Conversely, he described that the posterior triangle is the commonest site in head and neck to be involved, likely due to the small sample size in the present study.

At the current study, two out of 26 patients were diagnosed as LMs. Only one (50\%) case showed a trans-spatial mass, while the other one was limited to one space (submandibular space). This disagreed with Mahady et al, ${ }^{(17)}$ who described LMs as transspatial masses.

In this study, one out of 26 patients presented with lingual artery pseudoaneurysm that occurred secondary to hemi-mandibulectomy. This agreed with Schechter et al, ${ }^{(18)}$ who reported that lingual artery aneurysms are rare, eventhough their prevalence is unknown. It also agreed with van Cruijsen et $\mathrm{al},{ }^{(19)}$ who stated that most false aneurysms of the lingual artery have a traumatic or surgical origin.

In the present study, one out of 26 patients presented with neurofibroma, which matched Munir $\mathrm{N}$ et al., ${ }^{(20)}$ who stated that SM neural sheath tumors are rare and often simulate primary or metastatic disease. The patient presenting with neurofibroma in this study was a female agreeing with Luciana Sassa
Marocchio et al, ${ }^{(21)}$ who noted female predominance in cases with neurofibromas.

Luciana Sassa Marocchio et al, ${ }^{(21)}$ also stated in their own study that the real frequency of isolated neurofibromas unassociated with neurofibromatosis in the oral cavity is uncertain, but these tumors have been detected in the tongue, gingiva, palate, major salivary glands, and maxillary bones. Similarly in the present study, neurofibromas were noted in the SM and SL spaces. However, they were noted at the masticator and buccal spaces, retroauricular and subcutaneously at the parietal region as well.

In this study, two out of six FOM malignant lesions were diagnosed as squamous cell carcinoma of the FOM. Both were seen involving the anterior aspect of the FOM, specifically the SL space. This was consistent with Million RR et al, ${ }^{(22)}$ who reported that ninety percent of SCCs originate within $2 \mathrm{~cm}$ of the anterior midline FOM. ${ }^{(23)}$

In FOM carcinomas, no intra-glandular lymphatic metastasis was observed in this study. This went along with Cassie Fives et al, ${ }^{(24)}$ who described in a study that lymph nodes are not ordinarily present within the capsule of the SM gland, and thus, intraglandular lymphatic metastases are extremely unlikely from FOM carcinomas. The most common mechanism of SM gland involvement by oral cancer would appear to be direct extension and infiltration, usually from the primary tumour, or less commonly from an adjacent metastatic lymph node, this was again in agreement with Spiegel et al, ${ }^{(25)}$ whose study showed SM gland involvement in only $4 \%$ of FOM cancer.

Ganeshan et al, ${ }^{(26)}$ mentioned in their own study that the single best discriminator favoring malignancy was ill-defined margins. This agreed with our study, as five out of six $(83.3 \%)$ patients with malignant lesions had ill-defined borders. In another study, Balaji Ganeshan, et al, ${ }^{(26)}$ described how heterogeneity is a well-recognized feature of malignancy. This was similar to the present study, in which all malignant neoplastic lesions showed heterogeneous post-contrast enhancement. 
Firat et al, ${ }^{(27)}$ concluded in his study that benign non-neoplastic cysts were the most frequent lesions (40\%), purulent material consistent with active inflammation was the second most common (35\%), and in $20 \%$ of patients were interpreted as neoplastic. This coincides with our current study, as five $(100 \%)$ cases that presented with purely cystic lesions were found to be benign lesions.

\section{CONCLUSION}

CT is within reach with rapid image acquisition; this is why it is a primary investigation to broadly differentiate between different pathological processes in the floor of the mouth. Based upon the MDCT characteristics of the lesion such as tissue density, tissue composition, enhancement pattern and vascularity, MDCT helped to narrow the differential diagnosis.

CT provides key information for proper staging of patients with oral cancer, such as the invasion depth, assessing if there is bone invasion and evaluation of lymph nodes.

Regarding sialolithiasis, MDCT can adequately assess the number of stones, exact location, associated sialectasia as well as sialadenitis, if present.

Limitations of the study were small sample size relative to the wide range of pathologies so we recommend large scale studies.

\section{REFERENCES}

1. Mohammad Reza Hayeri, Pouya Ziai, Monda L. Shehata, Oleg M. Teytelboym, and Brady K. Huang RadioGraphics 2016 36:6, 1888-1910

2. La'porte SJ, Juttla JK, Lingam RK. Imaging the floor of the mouth and the sublingual space. Radiographics 2011; 31(5):1215-30.

3. Carlson ER, Ord RA. Benign Pediatric Salivary Gland Lesions. Oral Maxillofac Surg Clin North Am 2016; 28(1):67-81.
4. Malard, O., Toquet, C., Jegoux, F., Bordure, P., Beauvillain de Montreuil, C., \& Gayet-Delacroix, M. (2004). Computed tomography in TN stage evaluation of oral cavity and oropharyngeal cancers. Clinical imaging, 28(5), 360-367. https://doi.org/10.1016/S0899-7071(03)00207-9

5. Yuan Y, Jiang M, Wu L, Tao X. Differential diagnostic value of diffusion-weighted and dynamic contrast-enhanced MR imaging in non-cystic lesions in floor of the mouth. Dentomaxillofac Radiol 2019; 48(3):20180240.

6. Ahmed S, Yousif OY, Abuzeid M. Tumours of Salivary Glands in Sudan. Inter J Otorhinolaryngology 2018; 5(1):5.

7. Rega AJ, Aziz SR, Ziccardi VB. Microbiology and antibiotic sensitivities of head and neck space infections of odontogenic origin. J Oral Maxillofac Surg 2006; 64(9):1377-80

8. Charnoff SK, Carter BL. Plunging ranula: CT diagnosis. Radiology 1986; 158(2):467-8.

9. Gupta A, Karjodkar FR. Plunging Ranula: A Case Report. Int Scholar Res Network 2011; 2011:806928.

10. Coit WE, Harnsberger HR, Osborn AG, Smoker WR, Stevens MH, Lufkin RB. Ranulas and their mimics: CT evaluation. Radiology 1987; 163(1):211-6.

11. Laine FJ, Smoker WR. Oral cavity: anatomy and pathology. Semin Ultrasound CT MR 1995; 16(6):527-45.

12. Madani G, Beale T. Inflammatory conditions of the salivary glands. Semin Ultrasound CT MR 2006; 27(6): 440-51.

13. La'porte SJ, Juttla JK, Lingam RK. Imaging the floor of the mouth and the sublingual space. Radiographics 2011; 31(5):1215-30

14. Harnsberger HR. Diagnostic imaging. Head and neck. Salt Lake City: Amirsys; 2004.

15. Meesa IR, Srinivasan A. Imaging of the oral cavity. Radiol Clin North Am 2015; 53(1):99-114.

16. Navarro OM, Laffan EE, Ngan BY. Pediatric soft-tissue tumors and pseudo-tumors: MR imaging features with pathologic correlation: part 1. Imaging approach, pseudotumors, vascular lesions, and adipocytic tumors. Radiographics 2009; 29(3):887-906.

17. Mahady K, Thust S, Berkeley R, Stuart S, Barnacle A, Robertson F, et al. Vascular anomalies of the head and neck in children. Quant Imaging Med Surg 2015; 5(6):886-97. 
18. Brindle RS, Fernandez PM, Sattenberg RJ, Flynn MB, Heidenreich JO. Idiopathic Lingual Artery Aneurysm: CT Findings and Endovascular Therapy: A Case Report. Interv Neuroradiol 2010; 16(1):103-6.

19. van Cruijsen N, Gravendeel J, Dikkers FG. Severe delayed posttonsillectomy haemorrhage due to a pseudoaneurysm of the lingual artery. Eur Arch Otorhinolaryngol 2008; 265(1): 115-7.

20. Munir N, J.Bradley P. Diagnosis and management of neoplastic lesions of the submandibular triangle. Oral Oncology $2008 ; 44(3)$

21. Marocchio LS, Oliveira DT, Pereira MC, Soares CT, Fleury RN. Sporadic and multiple neurofibromas in the head and neck region: a retrospective study of 33 years. Clin Oral Investig 2007; 11(2):165-9.

22. Million RR, Cassisi NJ, Mancuso AA. Oral cayit. In: Million RR, Cassisi NJ (eds). Management of head and neck cancer: a multidisciplinary approach. $2^{\text {nd }}$ ed. Philadelphia, Pa: Lippincott; 1994. 321-400.

23. Sigal R, Zagdanski AM, Schwaab G, Bosq J, Auperin A, Laplanche A, et al. CT and MR imaging of squamous cell carcinoma of the tongue and floor of the mouth. Radiographics 1996; 16(4):787-810.

24. Fives C, Feeley L, Sadadcharam M, O'Leary G, Sheahan P. Incidence of intraglandular lymph nodes within submandibular gland, and involvement by floor of mouth cancer. Eur Arch Otorhinolaryngol 2017; 274(1):461-6.

25. Spiegel JH, Brys AK, Bhakti A, Singer MI. Metastasis to the submandibular gland in head and neck carcinomas. Head Neck 2004; 26(12):1064-8.

26. Ganeshan B, Miles KA. Quantifying tumour heterogeneity with CT. Cancer Imaging 2013; 13(1):140-9.

27. Firat P, Ersoz C, Uguz A, Onder S. Cystic lesions of the head and neck: cytohistological correlation in 63 cases. Cytopathology 2007; 18(3):184-90. 